\title{
Mucoepidermoid carcinoma associated with acute lymphoblastic leukemia
}

\author{
John J. Zappia ${ }^{1}$ and Kenneth D. McClatchey ${ }^{2}$ \\ Departments of ${ }^{1}$ Otolaryngology and ${ }^{2}$ Pathology, Unicersity of Michigan, Ann Arbor, MI 48109 (U.S.A.) \\ (Received 1 February 1991) \\ (Accepted 11 April 1991)
}

Key words: Leukemia; Second malignancy; Parotid carcinoma

\begin{abstract}
As treatment and survival of acute leukemia as well as other types of pediatric cancer improve, the number of second malignancies in these treated patients will increase. Occurrence of these second malignancies in the head and neck is not frequently reported although it is known that radiation treatment, chemotherapy and sometimes the primary lesion itself are risk factors for neoplasia. Malignancies of the parotid gland in the pediatric population are known to be unusual. We discuss a mucoepidermoid carcinoma in a 10-year-old female who 7 years earlier had undergone successful treatment of acute lymphoblastic leukemia.
\end{abstract}

\section{Introduction}

Parotid tumors in the pediatric age group are unusual [2,3]. Among pediatric parotid tumors, mucoepidermoid carcinoma is a more common malignant tumor than in adults [5], where it comprises 3-10\% of parotid masses [1]. Some authors have found that mucoepidermoid carcinoma in children represents as much as $50 \%$ of all parotid tumors [2,3]. In a large series of pediatric patients followed after treatment for a malignancy, it has been observed that adult-type tumors, such as carcinoma, tend to appear earlier [9]. In this report a patient is discussed who presented with a mucoepidermoid carcinoma of the parotid gland 7 years after treatment of leukemia.

Correspondence: K.D. McClatchey, Department of Pathology, University of Michigan, Ann Arbor, MI 48109 , U.S.A. 


\section{Case report}

A 10-year-old female presented with a two-month history of a right parotid mass that had been refractory to antibiotic therapy. There was no associated pain, fever, erythema, trismus or facial nerve deficit.

Her past medical history was significant for acute lymphoblastic leukemia diagnosed at the age of 3 years. She had received combination chemotherapy (vincristine, 6-mercaptopurine, methotrexate, prednisone) for 3.5 years and had been in complete remission since initial induction.

At the time of examination, a soft non-tender $2.5 \mathrm{~cm}$ mass was present in the parotid area. The VIIth nerve was noted to be completely intact and no other cervical masses were observed.

A CAT scan of the parotid region revealed a zone of increased attenuation in the superficial lobe of the right parotid with no evidence of deep lobe or parapharyngeal space extension (Fig. 1).

At the time of parotidectomy, the superficial lobe was noted to contain a $2 \times 2$ $\mathrm{cm}$ lesion, microscopically confirmed to be low grade mucoepidermoid carcinoma

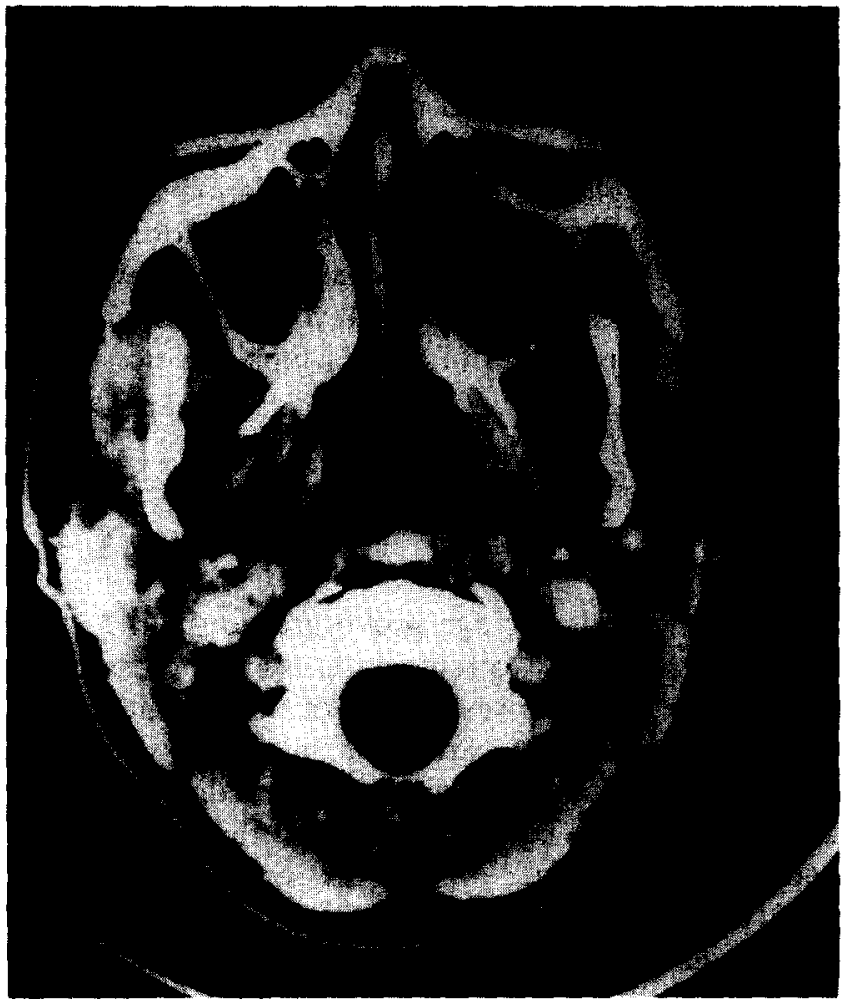

Fig. 1. A CAT scan of the parotid gland showed a zone of moderately increased attenuation in the lateral aspect of the superficial lobe of the right parotid gland which has poorly defined margins. There did not appear to be deep lobe involvement. 


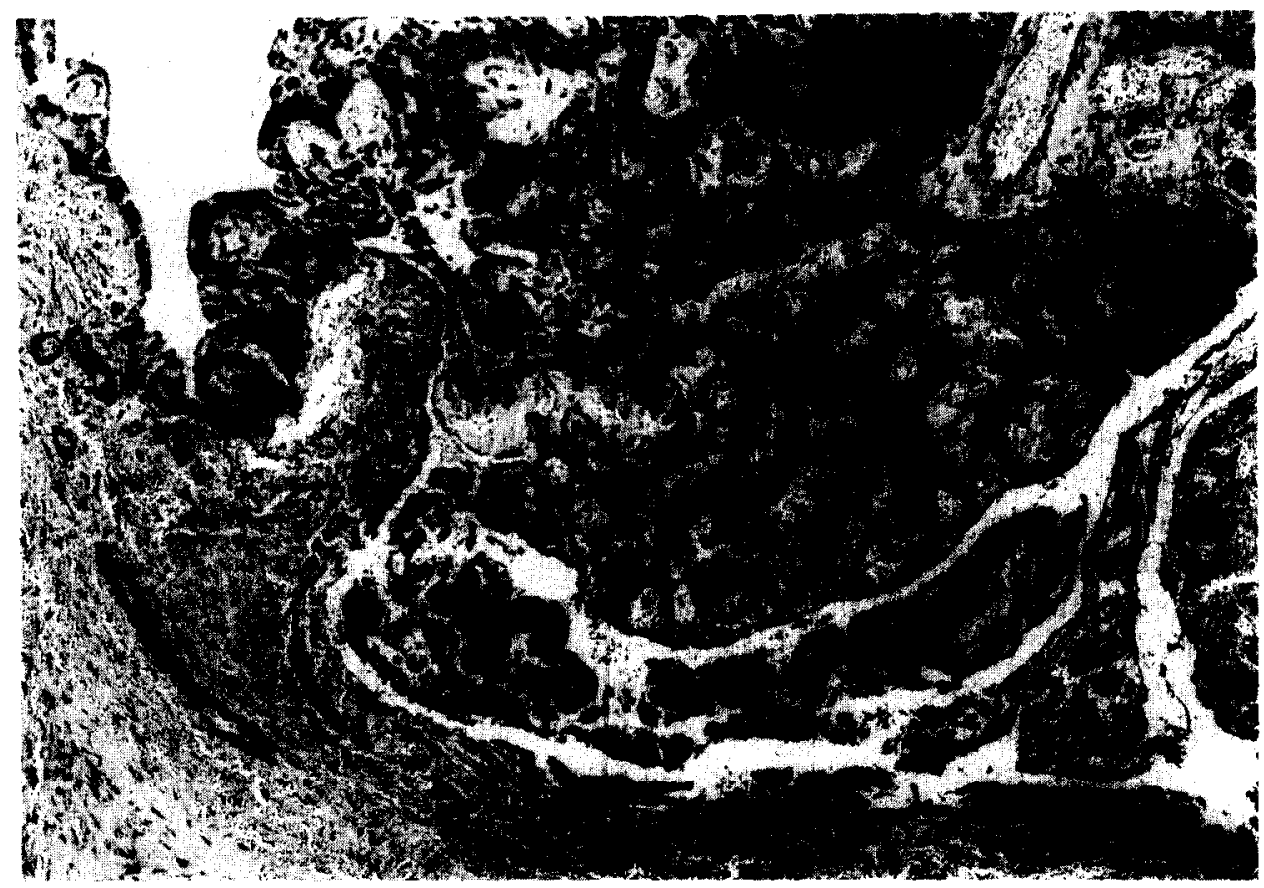

Fig. 2. Histopathologic evaluation of the parotid mass showed this low grade mucoepıdermoıd carcinoma with focal mucin (arrow). Note that the tumor is partially cystic. (mucicarmine stain; magnification $\times 300$ )

(Fig. 2). A total parotidectomy was performed. The postoperative course was uneventful. All of the branches of the VIIth nerve functioned fully. There has been no evidence of recurrence 24 months after surgery.

\section{Discussion}

The Late Effects Study Group (LESG), which has a large on-going multi-center study following children diagnosed with cancer, has found retinoblastoma is the most common first tumor in the patients that develop a second malignancy [10,11]. Other tumors, in decreasing order of frequency include, Hodgkin's disease, Wilm's tumor, soft tissue sarcomas, brain tumors, neuroblastomas and leukemia. Retinoblastoma and Hodgkin's disease typically make up $<10 \%$ of pediatric neoplasms but they represent $30 \%$ of primary tumors in this situation [11].

Kingston et al. [6], in their discussion of 161 pediatric patients with second primary neoplasms, report that central nervous system tumors are the most common first tumor $(45 / 161)$, retinoblastomas are the second $(37 / 161)$ and leukemia and lymphoma as a group comprise $33 / 161$. In their study they noted a change in pattern of first primaries. Prior to 1970 retinoblastoma and central nervous system tumors were most frequently associated with second primary 
tumors whereas after 1970 leukemia and lymphoma have been the major group. This may reflect improvement in survival of leukemia and lymphoma as well as exposure to more carcinogenic therapies.

When considering second primary tumors, osteosarcomas and chondrosarcomas are the most common [9]. Soft tissue and bone sarcomas compose 30\% of the second tumors [11]. Bone sarcomas are the most common second primary tumors in previously irradiated sites while leukemia is the most common cancer unassociated with radiation therapy [10]. It has also been noted that adult-type tumors, such as carcinomas, tend to appear at earlier ages [9].

In these patients, as well as in most other settings, radiotherapy is felt to be carcinogenic. Kingston et al. [6], in their review of pediatric patients with second neoplasms, found that 77 of 125 patients who received radiation had a second malignant neoplasm within the radiation field. Sixteen of these 125 patients developed leukemia. Overall they felt that they could attribute 93 of the $161(61 \%)$ second tumors to radiation. Meadows et al. [10] noted 208 of their $292(68 \%)$ pediatric patients had second malignant neoplasms developing in tissue exposed to radiation, with sarcoma being the most common. Hawkins et al. [4] noted a 6 -fold increase in the risk of second primary tumor with radiation and a 9-fold increase if chemotherapy is given in combination with radiation therapy.

The risk in patients receiving chemotherapy is less. In general the greatest risk in patients who received cytotoxic drugs was the development of leukemias and lymphomas [11]. Most larger studies do note an increased risk with cytotoxic drugs although not to the same degree as radiotherapy. Kingston et al. [6] noted that of 50 children who received cytoxic drugs the most common second primary tumor was acute non-lymphocytic leukemia.

Another risk factor in these patients is a genetic abnormality. This is known to be true for patients with retinoblastoma and at least partially explains why these patients make up such a large portion of the second maligancy group [10]. The role of heredity in the other first neoplasms is less certain.

The risk of a neoplasm in a child already treated for cancer is significantly higher than for age-matched controls. From 3 to $12 \%$ of these patients will develop a second tumor at least 3-5 years (depending on the methods in the particular study) after treatment of the initial cancer $[4,7,10,11,13]$. This represents a risk of $6-20 \times$ that of the control population.

The latency period between tumors varied from 5 months to 34 years in the experience of Meadows et al. [10]. When the tumor appeared within a radiation field the median latency was 10 years and when the second tumor was unassociated with radiation the median latency was 5 years. In our patient who did not receive radiation treatment the latency was 6 years.

In 1981, Mosijczuk and Ruymann [12] reviewed the literature and found 33 malignancies in patients who had been previously diagnosed with acute lymphoblastic leukemia. Nine of the secondary cancers were solid malignancies but none of the tumors were of the head and neck region.

In 1989, Loy et al. [8] reported two patients with acute leukemia who subsequently developed mucoepidermoid carcinoma of the parotid gland. Their first 
patient was a 15-year-old female with acute lymphoblastic leukemia who had been treated with combination chemotherapy as well as cranial radiation. Six years after diagnosis, and 3 years after completion of chemotherapy, a right parotid mucoepidermoid carcinoma was diagnosed. Their second patient was a 10 -year-old who had received combination chemotherapy and cranial radiation after diagnosis of acute myelomonocytic leukemia at age one year. This patient was treated with chemotherapy again at age 3 years for a relapse.

The only other reference to a parotid neoplasm occurring after treatment of a separate neoplasm was by Kingston et al. [6]. In one of their cases a carcinoma of the parotid gland occurred after treatment of leukemia but neither the type of leukemia nor the parotid cancer were identified.

The patient we presented is a 10 -year-old female who had been diagnosed with acute lymphoblastic leukemia at the age of 3 years. At that time she had been treated with a 3 -year course of combined chemotherapy with subsequent complete remission.

Tefft et al. [13] made the important statement concerning children that have been successfully treated for a primary malignancy - any child with a diagnosis of cancer, who survives the disease, must at no time be lost to follow-up examination at frequent intervals.

\section{References}

1 Batsakis, J.G. (Ed.), Tumors of the major salivary glands. In Tumors of the Head and Neck, Clinical and Pathological Considerations, Williams and Wilkins, Baltimore, 1979, pp. 34-39.

2 Byers, R.M., Piorkowski, R. and Luna, M.A., Malignant parotid tumors in patients under 20 years of age, Arch. Otolaryngol., 110 (1984) 232-235.

3 Castro, E.B., Huvos, A.G., Strong, E.S. and Foote, F.W., Tumors of the major salivary glands in children, Cancer, 29 (1972) 312-317.

1 Hawkins, M.M., Draper, G.J. and Kingston, J.E., Incidence of second primary tumours among childhood cancer survivors, Br. J. Cancer, 56 (1987) 339-347.

5 Jaques, D.A., Sigurds, S.O. and Chambers, R.G., Parotid tumors in children, Am. J. Surg., 132 (1976) 469-471.

6 Kingston, J.E., Hawkins, M.M., Draper, G.J., Marsden, H.B. and Kinnier Wilson, L.M., Patterns of multiple primary tumours of patients treated for cancer during childhood, Br. J. Cancer, 56 (1987) 331-338.

7 Li, F.P., Second malignant tumors after cancer in childhood, Cancer, 40 (1977) 1899-1902.

8 Loy, T.S., McLaughlin, R., Odom, L.F. and Dehner, L.P., Mucoepidermoid carcinoma of the parotid as a second malignant neoplasm in children, Cancer, 64 (1989) 2174-2177.

9 Meadows, A.T., D'Angio, G.J., Mike, V., Banfi, A., Harris, C., Jenkin, R.D.T. and Schwartz, A., Patterns of second malignant neoplasms in children, Cancer, 40 (1977) 1903-1911.

10 Meadows, A.T., Baum, E., Fossati-Bellani, F., Green, D. et al., Second malignant neoplasms in children: an update from the Late Effects Study Group, J. Clin. Oncol., 3 (1985) 532-538.

11 Meadows, A.T., Risk factors for second malignant neoplasms: report from the Late Effects Study Group, Bull. Cancer, 75 (1988) 125-130.

12 Mosijczuk, A.D. and Ruymann, F.B., Second malignancy in acute lymphocytic leukemia, Am. J. Dis. Child, 135 (1981) 313-316.

13 Tefft, M., Vawter, G.F. and Mitus, A., Second primary neoplas is in children, Am. J. Roentgenol., 103 (1968) 800-822. 